\title{
Primary Study Contact
}

National Cancer Institute

\section{Source}

National Cancer Institute. Primary Study Contact. NCI Thesaurus. Code C127533.

The main or principle contact person for a study. 